Gut and Liver, Vol. 10, No. 5, September 2016, pp. 842-850

\title{
Sensitization to and Challenge with Gliadin Induce Pancreatitis and Extrapancreatic Inflammation in HLA-DQ8 Mice: An Animal Model of Type 1 Autoimmune Pancreatitis
}

\author{
Sung-Hoon Moon ${ }^{1}$, Jihun Kim², Mi-Young Kim ${ }^{3}$, Do Hyun Park ${ }^{4}$, Tae Jun Song ${ }^{4}$, Sun A Kim², Sang Soo Lee ${ }^{4}$, Dong Wan \\ $\mathrm{Seo}^{4}$, Sung Koo Lee ${ }^{4}$, and Myung-Hwan $\mathrm{Kim}^{4}$ \\ ${ }^{1}$ Department of Internal Medicine, Hallym University Sacred Heart Hospital, Hallym University College of Medicine, Anyang, ${ }^{2}$ Department of \\ Pathology, Asan Medical Center, University of Ulsan College of Medicine, Seoul, ${ }^{3}$ Department of Internal Medicine, CHA Bundang Medical \\ Center, CHA University, Seongnam, and ${ }^{4}$ Department of Internal Medicine, Asan Medical Center, University of Ulsan College of Medicine, \\ Seoul, Korea
}

Background/Aims: The aim of this study was to establish a pathogenetic mechanism of pancreatitis in celiac disease and IgG4-related disease using gluten-sensitive human leukocyte antigen (HLA)-DQ8 transgenic mice. Methods: Transgenic mice expressing HLA-DQ8 genes were utilized. Control mice were not sensitized but were fed gliadin-free rice cereal. Experimental groups consisted of gliadin-sensitized and gliadin-challenged mice; nonsensitized mice with cerulein hyperstimulation; and gliadin-sensitized and gliadinchallenged mice with cerulein hyperstimulation. Results: Gliadin-sensitized and gliadin-challenged mice with cerulein hyperstimulation showed significant inflammatory cell infiltrates, fibrosis and acinar atrophy compared with the control mice and the other experimental groups. The immunohistochemical analysis showed greater IgG1-positive plasma cells in the inflammatory infiltrates of gliadin-sensitized and gliadin-challenged mice with cerulein hyperstimulation compared with the control mice and the other experimental groups. Gliadin-sensitized and gliadin-challenged mice with cerulein hyperstimulation or gliadin-sensitized and gliadinchallenged mice showed IgG1-stained inflammatory cell infiltrates in the extrapancreatic organs, including the bile ducts, salivary glands, kidneys, and lungs. Conclusions: Gliadinsensitization and cerulein hyperstimulation of gluten-sensitive HLA-DQ8 transgenic mice resulted in pancreatitis and extrapancreatic inflammation. This animal model suggests that chronic gliadin ingestion in a susceptible individual with the HLA-DQ8 molecule may be associated with pancreatitis and extrapancreatic inflammation. (Gut Liver 2016;10:842850)

Key Words: Autoimmune diseases; Pancreatitis; Celiac disease

\section{INTRODUCTION}

Celiac disease (CD) is an immune-mediated enteropathy with gluten sensitivity that is associated with major histocompatibility complex (MHC) II antigens, specifically human leukocyte antigen (HLA)-DQ2 and HLA-DQ8. ${ }^{1,2}$ CD is also associated with various systemic autoimmune diseases, including Addison's disease, autoimmune thyroid disease, autoimmune insulindependent diabetes mellitus, Sjögren syndrome, primary biliary cirrhosis, and autoimmune hepatitis. ${ }^{2}$ Moreover, CD is associated with chronic pancreatitis, ${ }^{3-12}$ and a population-based cohort study from Sweden showed that patients with CD had an almost 3-fold higher risk of developing pancreatitis than the general population. ${ }^{8}$ However, little is known about the potential mechanisms underlying the association between CDs and pancreatitis.

Sensitization of HLA-DQ8 transgenic mice to gliadin, which is a water soluble component of gluten, may induce pancreatic inflammation. HLA-DQ8 transgenic mice express the human DQ8 molecule, but lack endogenous murine class II molecules. The administration of an anti-CD25 monoclonal antibody to deplete CD25+Foxp3+T cells before sensitization to gliadin induces severe insulitis in nonobese diabetic (NOD)-DQ8 mice. ${ }^{13}$

Correspondence to: Myung-Hwan Kim

Department of Internal Medicine, Asan Medical Center, University of Ulsan College of Medicine, 88 Olympic-ro 43-gil, Songpa-gu, Seoul 05505, Korea

Tel: +82-2-3010-3180, , Fax: +82-82-2-485-5782, E-mail: mhkim@amc.seoul.kr

Received on September 24, 2015. Revised on October 27, 2015. Accepted on November 10, 2015. Published online April 28, 2016 pISSN 1976-2283 eISSN 2005-1212 http://dx.doi.org/10.5009/gnl15484

Sung-Hoon Moon and Jihun Kim contributed equally to this work as first authors.

@. This is an Open Access article distributed under the terms of the Creative Commons Attribution Non-Commercial License (http://creativecommons.org/licenses/by-nc/4.0) which permits unrestricted non-commercial use, distribution, and reproduction in any medium, provided the original work is properly cited. 
Furthermore, increased tissue transglutaminase activity in the pancreas of 12-week-old NOD mice showed ongoing pancreatic inflammation, ${ }^{14}$ suggesting that sensitization of DQ8 mice to gliadin may induce pancreatic injury.

Autoimmune pancreatitis (AIP) is a form of chronic pancreatitis characterized by an autoimmune inflammatory process. AIP was first described in $1961^{15}$ and proposed as a distinct clinical entity in $1995 .{ }^{16}$ Clinically, type 1 AIP is characterized by elevated serum titers of IgG4, lymphoplasmacytic infiltrates around the pancreatic ducts, acinar lymphoplasmacytic inflammation with atrophy and fibrosis, and obliterative phlebitis. ${ }^{17}$ Systemic manifestations include infiltration of the gallbladder, bile ducts, kidneys, lungs, and salivary glands by lymphocytes and IgG4-positive plasma cells. Like AIP, CD patients with chronic pancreatitis are also known to have elevated serum concentrations of IgG. ${ }^{18}$

We therefore hypothesized that chronic pancreatitis in DQ8 mice may be similar, serologically, histologically, and immunohistochemically, to human type 1 AIP, based on the premise that mouse IgG1 is a homologue of human IgG4. The aim of our study was to establish a pathogenetic mechanism of pancreatitis in $\mathrm{CD}$ and IgG4-related disease using gluten-sensitive HLA-DQ8 transgenic mice.

\section{MATERIALS AND METHODS}

\section{Mice}

Transgenic mice expressing HLA-DQ8 (CD2-CD4, HLADQA1*0301/HLA-DQB1*0302) genes were purchased from The Jackson Laboratory (Bar Harbor, ME, USA) and were bred in the specific pathogen-free unit (temperature, $22^{\circ} \mathrm{C} \pm 2^{\circ} \mathrm{C}$; humidity, $60 \% \pm 4 \%$ ) with a 12 -hour light/dark cycle at the Animal Resource Center at the Asan Institute for Life Science. The mice were maintained at least two generations before breeding on a gluten-free diet (Jaden Bioscience, San Diego, CA, USA). All mice were genotyped using polymerase chain reaction protocols provided by the Jackson Laboratory and were used at the age of 10 to 30 weeks. C57BL/6 mice were purchased from Orient Bio (Seongnam, Korea).

All mice used in this experiment were cared for and were handled humanely following the NIH Principles of Laboratory Animal Care (U.S. NIH publication number: 85-23, revised 1985) and the guidelines for animal experiments at the Asan Institute for Life Sciences and Technology. This animal study was approved by our institutional animal care and use committee.

\section{In vivo experimental groups}

To induce pancreatitis in mice, we injected cerulein to peritoneal cavity. Cerulein, a decapeptide that acts on the cholecystokinin receptor, is known to induce pancreatitis by hyperstimulation of exocrine pancreas. For the cerulein hyperstimulation, sixteen C57BL/6 mice were injected intraperitoneally with 50 $\mu \mathrm{g} / \mathrm{kg}$ of cerulein (dissolved in sterile water) on 7 consecutive hours, twice a week for 10 weeks, and once a week for 10 additional weeks (a total of 20 weeks).

To establish experimental model for $\mathrm{CD}$, we challenged gliadin-sensitized HLA-DQ8 mice with gliadin feeding. All HLADQ8 transgenic mice were continuously fed with gliadin-free diet before experiments. We also used combined intraperitoneal cerulein injection to augment pancreatitis. Thirty-two transgenic DQ8 mice were divided into four different groups: (1) nonsensitized mice injected with Complete Freund's Adjuvant (CFA; Sigma-Aldrich, St. Louis, MO, USA) only, and fed with gliadinfree rice cereal (2 mg/0.02 mM acetic acid) ( $\mathrm{n}=8)$; (2) gliadinsensitized mice subsequently challenged with oral gliadin (2 $\mathrm{mg} / 0.02 \mathrm{mM}$ acetic acid) ( $\mathrm{n}=8)$; (3) nonsensitized mice injected intraperitoneally with cerulein (50 $\mu \mathrm{g} / \mathrm{kg})(\mathrm{n}=8)$; and (4) gliadinsensitized mice subsequently challenged with oral gliadin $(2$ $\mathrm{mg} / 0.02 \mathrm{mM}$ acetic acid) and intraperitoneal cerulein (50 $\mu \mathrm{g} / \mathrm{kg}$; American Peptide Co., Sunnyvale, CA, USA) (n=8).

For sensitization, mice were injected intraperitoneally with $500 \mu \mathrm{g}$ of gliadin (gliadin from wheat; Sigma-Aldrich, Oakville, Canada) dissolved in $0.02 \mathrm{mM}$ acetic acid in $50 \mu \mathrm{g}$ of CFA. After the first week, gliadin challenge was performed $3 \times /$ week by intragastric gavage using $2 \mathrm{mg}$ of gliadin dissolved in $0.02 \mathrm{mM}$ acetic acid. Intraperitoneal cerulein injection was performed with $50 \mu \mathrm{g} / \mathrm{kg}$ of cerulein (dissolved in sterile water) on 7 consecutive hours, twice a week for 10 weeks, and once a week for 10 additional weeks. All mice were continuously fed a gliadinfree rice cereal. The mice were killed 24 hours after the last oral challenge and tissues were observed by hematoxylin and eosin (H\&E) stain.

\section{Immunohistochemistry on harvested mouse tissue}

Formalin-fixed organ samples were paraffin-embedded and sections were stained by H\&E for histology. For immunohistochemistry, tissue sections were deparaffinized in xylene and were rehydrated through a series of alcohols and distilled water. Epitope retrieval was performed in preheated antigen unmasking solution (Vector Laboratories, Burlingame, CA, USA) in a water bath. Three percent peroxidase-blocking reagent was applied for 5 minutes. Sections were stained using polyclonal goat antimouse IgG1 (dilution 1:2000, A90-105A; Bethyl Laboratories, Montgomery, TX, USA) and biotinylated anti-goat secondary antibody (Vector Laboratories, pk-6105). The detection system consisted of VECTASTAIN ABC reagent (Vector Laboratories, pk-6105) with DAB chromogen (Dako, Glostrup, Denmark). Sections were washed with phosphate-buffered saline three times among each incubation. Sections were counterstained with hematoxylin (Vector Laboratories, H-3401). 


\section{Mouse lgG1 measurements in serum}

Mouse IgG1 is a homologue of human IgG4. ${ }^{19,20}$ Blood samples were obtained from the ophthalmic plexus of each group of mice. Sera were obtained after centrifugation at 3,000 rpm for 10 minutes in room temperature and stored at $-70^{\circ} \mathrm{C}$ until assayed. IgG1 levels in sera were quantified using ELISA kit (Immunology Consultants Laboratory, Portland, OR, USA) according to the manufacturer's instructions.

\section{Histologic analysis and semiquantification of pancreatitis}

Pancreatitis was graded according to a 3-tier scoring system in terms of acinar inflammation, acinar atrophy, ductal inflammation, and stromal fibrosis. Scoring system was adopted from the histological score for chronic pancreatitis described by Demols et al. ${ }^{21}$ with slight modification. Specifically, acinar inflammation and acinar atrophy were scored by percentage of involved area: absent as $0,<10 \%$ of lobule as $1,10 \%$ to $50 \%$ of lobule as 2 , and $>50 \%$ of lobule as 3 . Ductal inflammation was scored as follows: absent as 0 , patchy and mild as 1 , dense as 2 , and ductal destruction as 3. Stromal fibrosis was scored by percentage of fibrous tissue in examined tissue section; absent as $0,<10 \%$ as $1,10 \%$ to $50 \%$ as 2 , and $>50 \%$ as 3 . Total score of 0 to 3 was classified as mild, 4 to 6 as moderate, and 7 to 9 as severe. Scoring was done by two independent pathologists (J.K., S.A.K.) in a blinded manner and any discrepancy was resolved by consensus. The number of immunohistochemically identified IgG1-positive plasma cells in pancreas were counted in each specimen per high-power field. Positive IgG1 immunostaining was defined as more than 10 IgG1-positive plasma cells in at least 1 high-power field at a magnification of $\times 400$.

\section{Statistical analysis}

Data were analyzed with Prism 5 Software (GraphPad, La
Jolla, CA, USA). Categorical parameters were compared by the use of the chi-square test or the Fisher exact test, and continuous variables were compared by using the Student t-test or the Mann-Whitney U test. A p-value $<0.05$ was considered statistically significant.

\section{RESULTS}

\section{Intraperitoneal cerulein injection alone did not induce significant pancreatitis in C57BL/6 mice}

In C57BL/6 mice with cerulein hyperstimulation, only mild nonspecific inflammatory cell infiltration was seen in the pancreas (Fig. 1) compared with control C57BL/6 mice. No definite acute or chronic pancreatitis was observed.

\section{Gliadin sensitization and challenge in HLA-DQ8 transgenic mice invoked the production of anti-gliadin antibodies}

Gliadin-sensitized HLA-DQ8 (CD2-CD4, HLA-DQA1*0301/ HLA-DQB $1{ }^{*} 0302$ ) transgenic mice did not show significant poor growth or a wasted appearance after subsequent oral challenge of gliadin (2 mg/0.02 mM acetic acid) for 35 weeks. Histologically, no significant villous blunting or intraepithelial lymphocytosis, which is histologic hallmark of $\mathrm{CD}$, was observed in the small intestine although mononuclear inflammatory cell infiltration was mildly increased and villous height was mildly decreased compared to controls (Fig. 2). However, serum antigliadin IgG was highly elevated in the gliadin-sensitized and -challenged mice while it was not changed in unchallenged controls. The degree of pancreatitis was positively associated with the level of anti-gliadin IgG in the sera (data not shown). However, anti-gliadin IgA was not detectable in sera of any mice.
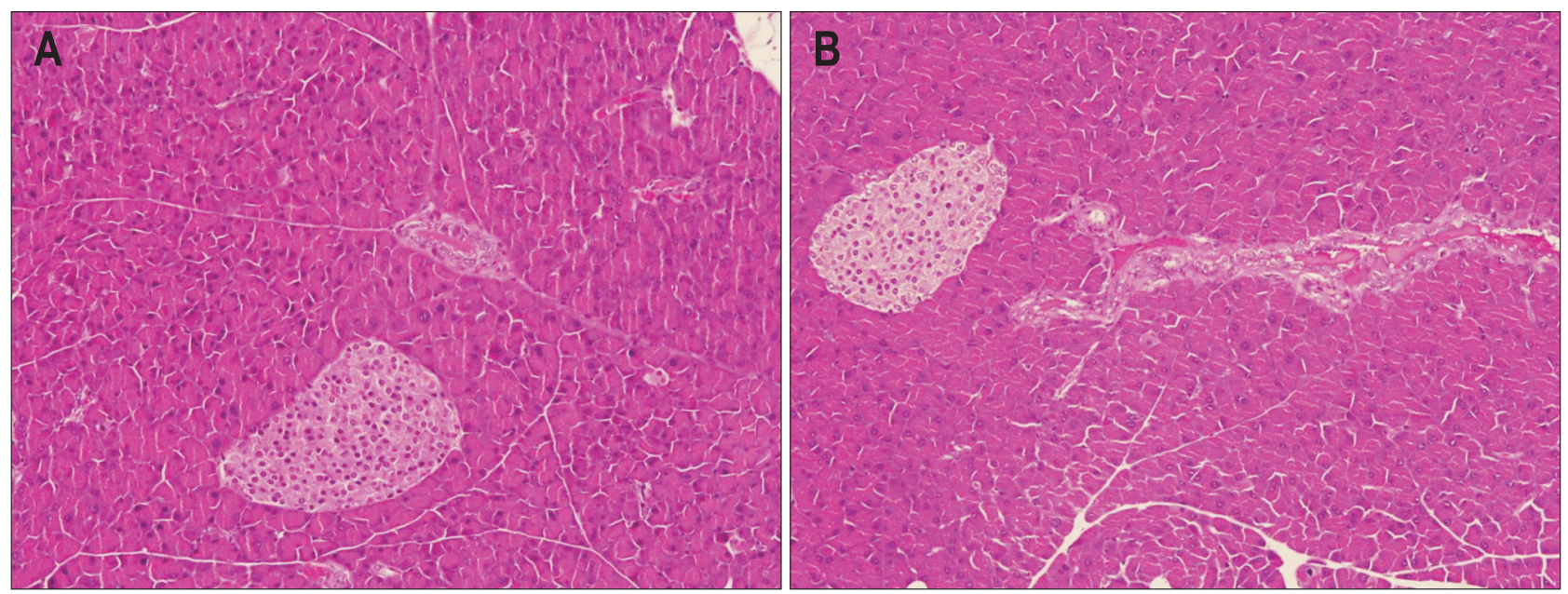

Fig. 1. Histology of the pancreas of C57BL/6 mice with or without intraperitoneal cerulein injection. No significant inflammation was observed in either the mock-treated (A) or the cerulein-injected (B) C57BL/6 mice (H\&E stain, $\times 200)$. 

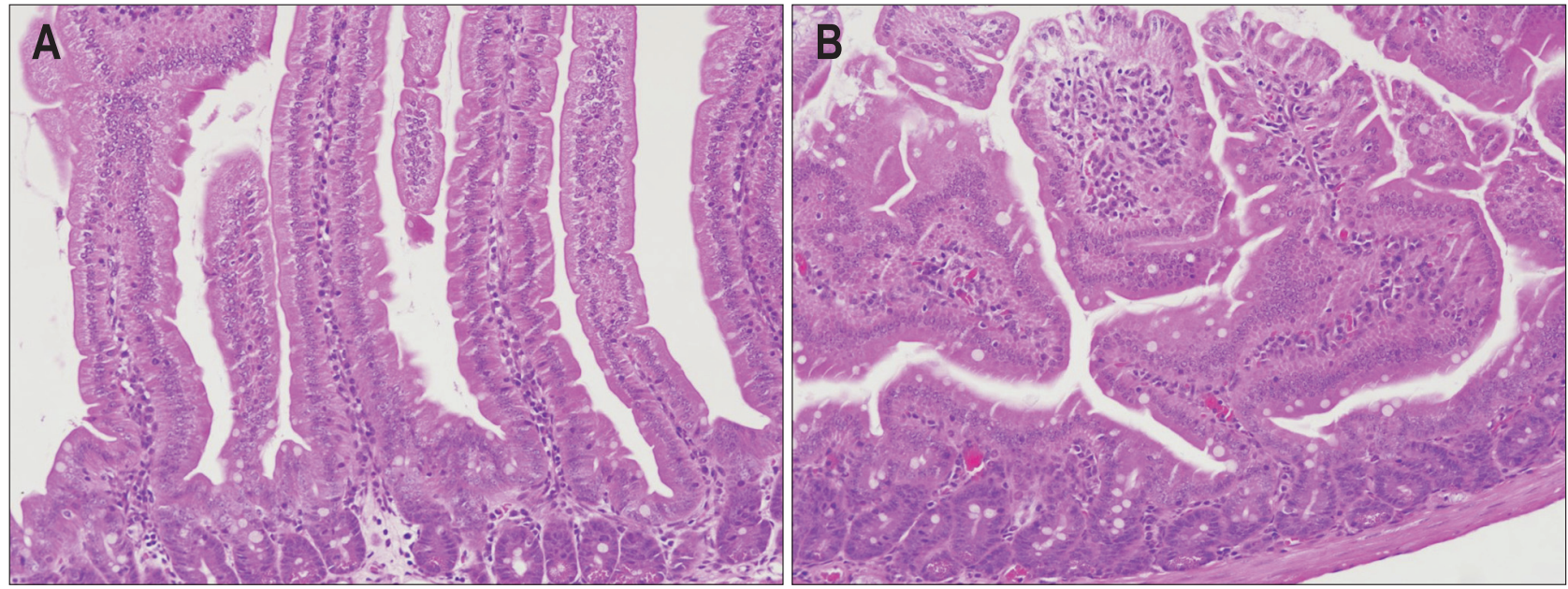

Fig. 2. Histology of the proximal small intestinal mucosa of human leukocyte antigen (HLA)-DQ8 mice. (A) Long villi are well-preserved in mice fed a gliadin-free diet. (B) Mild villous blunting and increased lymphoplasmacytic infiltration are observed in gliadin-sensitized and gliadinchallenged mice (H\&E stain, $\times 200)$.

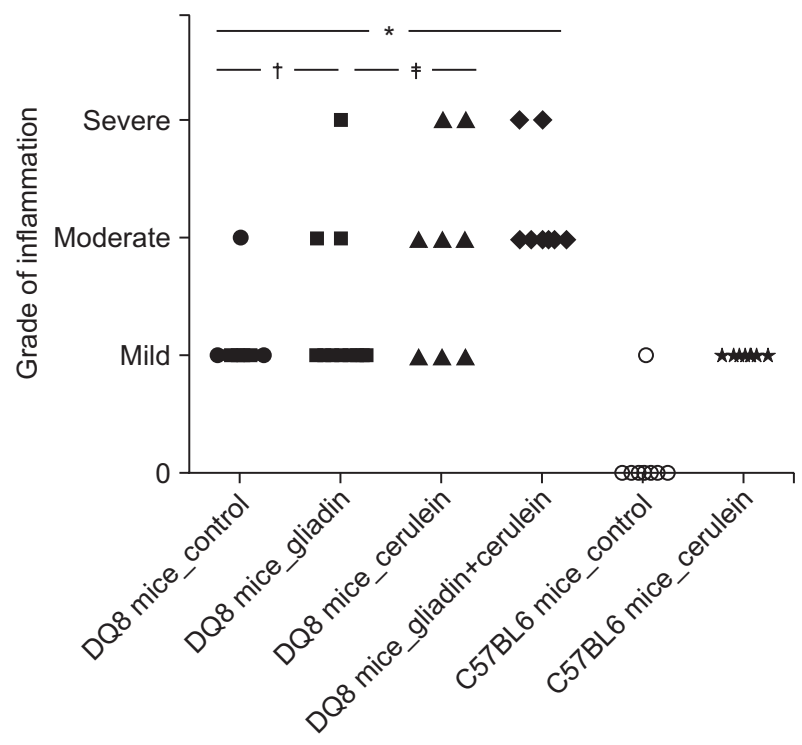

Fig. 3. Semi-quantification of pancreatic inflammation. Human leukocyte antigen (HLA)-DQ8 mice with exposure to both gliadin and cerulein have significantly higher inflammation scores than those undergoing the mock treatment $\left({ }^{*} \mathrm{p}=0.002\right)$, gliadin-only exposure $\left({ }^{\dagger} \mathrm{p}=0.032\right)$, or cerulein-only exposure $(\mathrm{p}=0.049)$. No significant difference was observed between the gliadin-only treatment group and the cerulein-only treatment group $\left({ }^{\ddagger} \mathrm{p}=0.674\right)$.

\section{Pancreatitis was most robustly induced when HLA-DQ8 mice were subjected to gliadin sensitization/challenge and cerulein hyperstimulation at the same time}

To examine whether pancreatitis is induced in our mouse CD model and whether the pancreatitis pathologically mimics human AIP, we analyzed pancreatic tissue specimen of mice treated with various combinations of gliadin sensitization/challenge and intraperitoneal cerulein injection. Pancreatitis was best induced when HLA-DQ8 mice were gliadin-sensitized and -challenged and were further stimulated by intraperitoneal injection of cerulein. In semiquantitative analyses, mice with both gliadin-sensitization/challenge and cerulein hyperstimulation showed significantly higher levels of inflammatory cell infiltrates, fibrosis and acinar atrophy than those with vehicle treatment $(p=0.002)$, gliadin-sensitization/challenge $(p=0.032)$, or cerulein hyperstimulation ( $p=0.049$ ) (Fig. 3). The degree of pancreatitis of gliadin treatment group was not different from that of cerulein hyperstimulation group. Histologically, pancreatitis in our model partially mimicked human type 1 AIP. Specifically, patchy infiltration of mixed inflammatory cell infiltration including lymphocytes, plasma cells, and neutrophils was observed throughout the entire pancreas. In the involved area, storiform fibrosis and acinar atrophy were observed (Fig. 4). Obliterative phlebitis, which is another histologic hallmark of human type 1 AIP, was not seen.

\section{IgG1 staining was proportional to the degree of pancreatitis in HLA-DQ8 mouse models}

Because histologic features of chronic pancreatitis in our model, especially gliadin-sensitized/challenged and cerulein hyperstimulated group, were similar to those of human AIP, we set out to examine the expression of IgG1, which is known as the mouse counterpart of human IgG4, ${ }^{19,20}$ in the various organs of our models. In gliadin-sensitized/challenged mice, numerous IgG1 stained plasma cells were seen in the involved pancreas parenchyma (Fig. 4). Moreover, IgG1-positive stromal cells, acinar cells and ductal cells were noted in the pancreatitis lesions, but not in areas of histologically normal parenchyma. In gliadin-sensitized/challenged and cerulein hyperstimulated mice, the number of IgG1-positive cells was significantly higher than those in vehicle treated controls, gliadin treated, or ceru- 

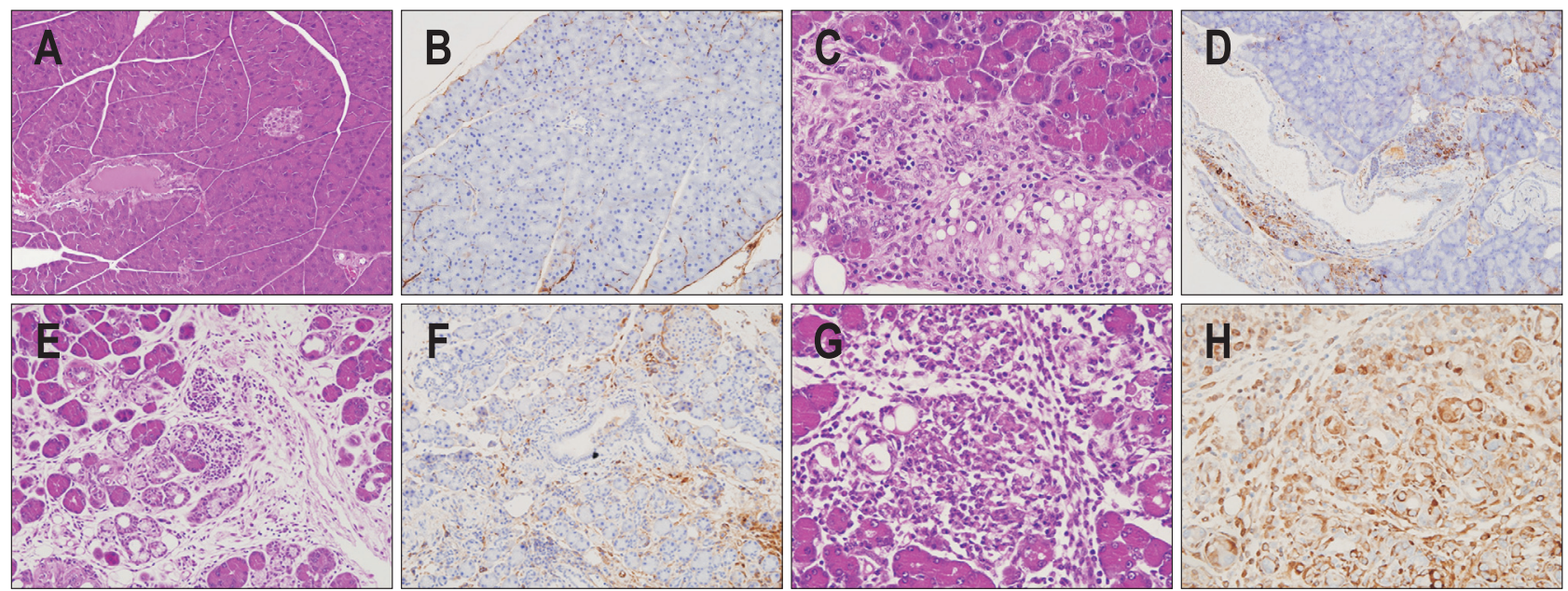

Fig. 4. Histology of the pancreases of human leukocyte antigen (HLA)-DQ8 mice with or without exposure to gliadin and/or cerulein. (A, B) Pancreases of gliadin-restricted mice look normal and do not exhibit IgG1-positive cells. (C, D) Pancreases of the gliadin-only group have inflammatory foci showing lymphoplasmacytic infiltration, fibrosis and lobular atrophy. Several IgG1-positive cells are observed. (E, F) Pancreases of the cerulein-only group exhibit focal mild inflammatory cell infiltration. Mild edema and fibrosis can also be seen, and there are several IgG1-positive cells. (G, H) Mice with exposure to both gliadin and cerulein show profound lymphoplasmacytic infiltration, acinar atrophy and storiform fibrosis. Many IgG1-positive plasma cells are observed, and certain acinar cells and stromal cells are stained by IgG1 (A, E: H\&E stain, ×100; C, G: H\&E stain, $\times 200$; B, D, F, and G: IgG1, ×100).

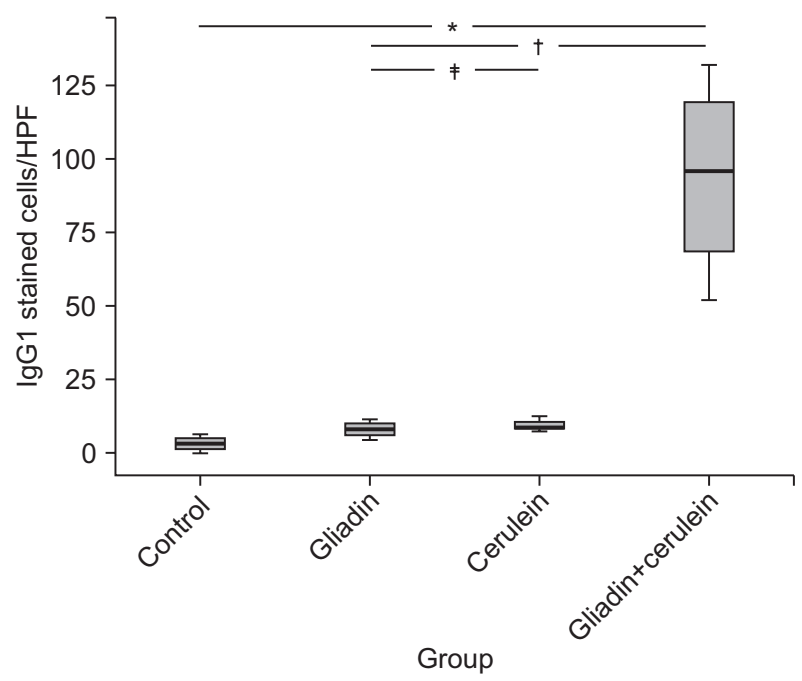

Fig. 5. Quantification of IgG1-positive plasma cells. Human leukocyte antigen (HLA)-DQ8 mice exposed to both gliadin and cerulein show a significantly higher number of IgG1-positive cells than the control, gliadin-only or cerulein-only mice $\left({ }^{*} \mathrm{p}=0.021,{ }^{\dagger} \mathrm{p}=0.034\right.$, and $\mathrm{p}=0.034$ ). There is no significant difference between the gliadin-only and cerulein-only groups $\left(^{\ddagger} \mathrm{p}=0.513\right)$.

HPF, high power field.

lein treated group ( $\mathrm{p}=0.021, \mathrm{p}=0.034$, and $\mathrm{p}=0.034$, respectively) (Figs 4 and 5). No significant difference was shown between gliadin-sensitized/challenged mice and mice with cerulein hyperstimulation. No significant IgG1-positive inflammatory cells were found on control mice.

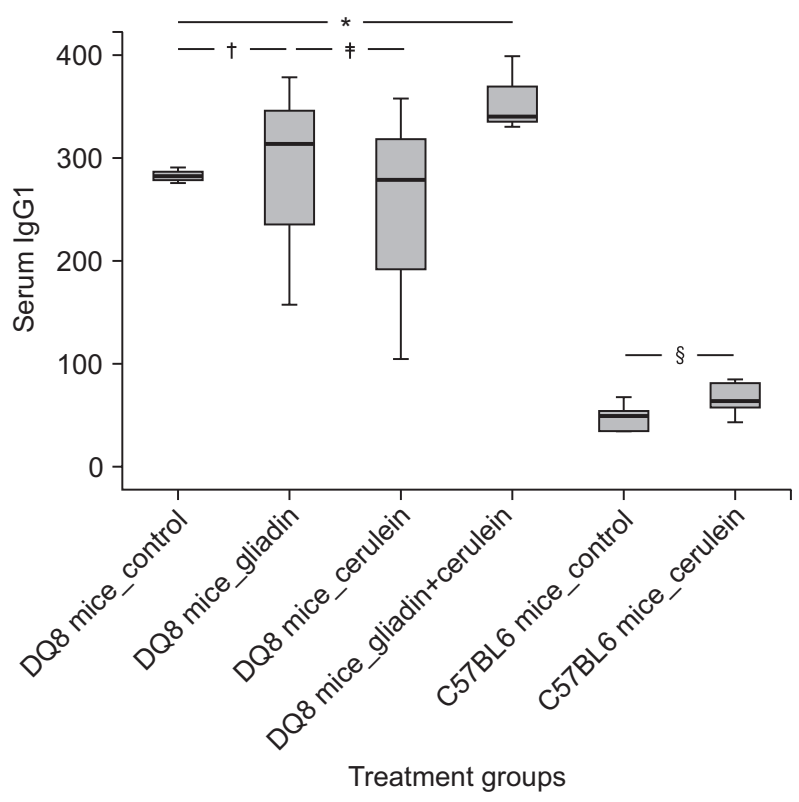

Fig. 6. Serum IgG1 levels. Human leukocyte antigen (HLA)-DQ8 mice exposed to both gliadin and cerulein show a significantly higher level of serum IgG1 than control mice ( $\mathrm{p} p=0.034)$. No differences are found among the control, gliadin-only, and cerulein-only groups ( ${ }^{\dagger} \mathrm{p}=0.513$ and $\left.{ }^{\ddagger} \mathrm{p}=0.513\right)$. C57BL/6 mice show lower levels of serum IgG1 than HLA-DQ8 mice regardless of cerulein injection $\left({ }^{\S} \mathrm{p}=0.116\right)$.

\section{Serum IgG1 was also elevated in HLA-DQ8 mouse models}

HLA-DQ8 transgenic mice with gliadin-sensitization/challenge and cerulein hyperstimulation showed significantly higher level of serum IgG1 than vehicle-treated HLA-DQ8 mice 
$(\mathrm{p}=0.034)$. Serum IgG1 levels of HLA-DQ8 mice with either gliadin or cerulein treatment alone were not different from those of vehicle-treated HLA-DQ8 mice. HLA-DQ8 transgenic mice had higher levels of serum IgG1 than C57BL6 mice, regardless of cerulein or gliadin treatment (Fig. 6).

\section{HLA-DQ8 transgenic mice with chronic pancreatitis frequently showed extrapancreatic inflammation like human type 1 AIP}

Among 16 gliadin-sensitized and -challenged mice with chronic pancreatitis, seven (44\%, 6-gliadin-sensitized and -challenged mice with cerulein hyperstimulation, one in gliadin-sensitized and -challenged mice) mice showed chronic inflammation of the same pattern as pancreatitis in various extrapancreatic organs including extrapancreatic bile duct $(n=4)$, salivary gland $(\mathrm{n}=1)$, kidney $(\mathrm{n}=1)$, and the lung $(\mathrm{n}=2)$ (Fig. 7). The extrapancreatic organ involvement was more frequent in combined gliadin and cerulein treatment group $(n=6)$ than in gliadin treatment alone group $(n=1)$. The degrees of inflammation in the involved extrapancreatic organs were more prominent in mice with severe pancreatitis than in those with mild to moderate pancreatitis (data not shown). Immunohistochemical staining also showed positive IgG1 stained plasma cells in the inflammatory cell infiltrates in the involved extrapancreatic organs (Fig. 8).
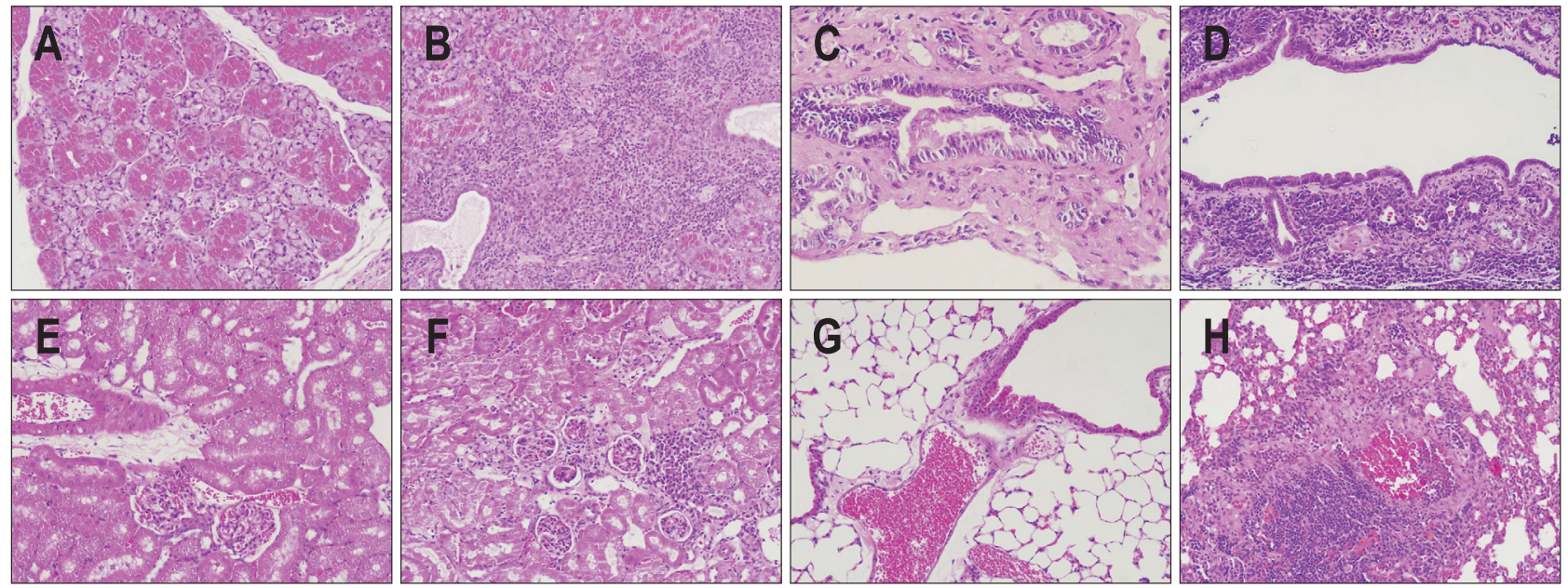

Fig. 7. Histology of selected extrapancreatic organs in both the control and gliadin/cerulein double-exposed human leukocyte antigen (HLA)-DQ8 mice. The salivary glands (A), extrapancreatic bile ducts (C), kidneys (E) and lungs (G) of the control mice do not show significant inflammation. In the gliadin and cerulein double-exposed mice, marked lymphoplasmacytic infiltration and fibrosis are observed in the salivary glands (B), extrapancreatic bile ducts (D), and lungs (H). The kidney (F) is mildly involved (H\&E stain, $\times 100)$.
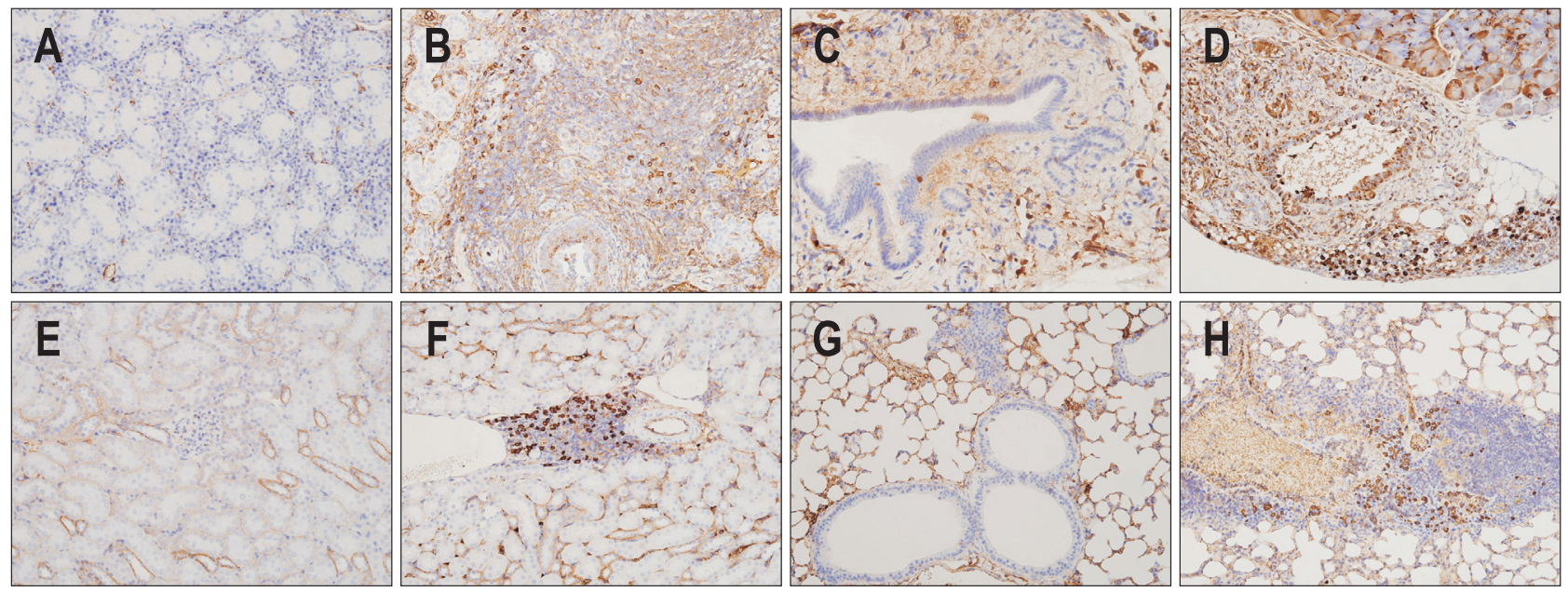

Fig. 8. IgG1 staining of selected extrapancreatic organs in both the control and gliadin/cerulein double-exposed human leukocyte antigen (HLA)DQ8 mice. In the control mice, no IgG1-positive plasma cells are observed, although there is a degree of cross reactivity in the endothelial cells, distal renal tubules, and lung alveoli (A, C, E, and G). In double-exposed mice, many IgG1-positive plasma cells are shown in the salivary glands (B), extrapancreatic bile ducts (D), kidneys (F), and lungs $(\mathrm{H})(\mathrm{IgG} 1, \times 100)$. 


\section{DISCUSSION}

In the current study, we successfully induced chronic pancreatitis, which is pathologically similar to human type 1 AIP, by gliadin sensitization/challenge and cerulein hyperstimulation in HLA-DQ8 transgenic mice. This model might explain a potential pathogenetic mechanism of chronic pancreatitis that occurs in the context of $\mathrm{CD}$. In $\mathrm{CD}$, malnutrition is known to impair the pancreatic secretion and structural changes, including acinar atrophy, in the pancreas. ${ }^{22}$ Villous atrophy is associated with pancreatic insufficiency, and restored pancreatic enzyme levels are observed after introduction of a gluten-free diet. ${ }^{23}$ Papillary stenosis due to duodenal inflammation may be also associated with pancreatitis. ${ }^{9}$ Besides malnutrition, villous atrophy, and papillary stenosis, the altered level of autoregulatory hormone (such as cholecystokinin) may contribute to the development of pancreatitis. $^{23}$ Moreover, in our DQ8 mice, nonspecific hyperstimulation of exocrine pancreas alone induced mild to moderate pancreatitis. This suggested that genetically susceptible individuals may be vulnerable to acute or chronic pancreatitis, irrespective of gliadin-sensitization and challenge, corroborating previous studies where both $\mathrm{CD}$ and pancreatitis showed increased Th1-associated cytokines. ${ }^{24-26}$ However, only 10\% of CD patients have DQ8, while 90\% of celiac patients have HLADQ2. ${ }^{27}$ This may be a possible reason why most patients with CD do not apparently show pancreatitis.

Although the pathogenesis of AIP remains to be elucidated, several reports suggested that susceptibility to AIP may be related to certain MHC class II allele. ${ }^{28,29}$ In the present study, we found that gliadin exposure in gluten-sensitive transgenic mice showed chronic pancreatitis similar to human AIP. Furthermore, additional cerulein hyperstimulation in this context induced far more severe pancreatitis. We also found that chronic pancreatitis in our model showed prominent expression of IgG1, the mouse homologue of human IgG4. ${ }^{19,20,30}$ In addition, we found that mice with both gliadin and cerulein exposures showed a strong IgG1 staining not only in the plasma cells but also in the stromal, acinar and ductal cells. We think that IgG1 staining in parenchymal cells other than plasma cells might represent active secretion of IgG1 rather than nonspecific staining as those parenchymal cells in control mice did not show such IgG1 staining. Furthermore, tissue $\operatorname{IgG} 1$ expression was positively associated with serum IgG1 level. Interestingly, gliadin-sensitization/challenge and subsequent cerulein hyperstimulation in HLA-DQ8 transgenic mice also showed chronic inflammation with abundant IgG1 expression in the extrapancreatic organs including the bile duct, salivary gland, kidney and lung. These data suggest that gluten-sensitive HLA-DQ8 transgenic mice with exogenous antigens may serve as an animal model of IgG4-related pancreatitis and extrapancreatic involvement.

Contrary to other studies with autoreactive T cells, ${ }^{17,29,31}$ we used gliadin as exogeneous antigens. To date, despite many efforts for the elucidation of autoantigen in AIP, little is known about specific autoantigen in AIP. Our results suggest that AIP with or without extrapancreatic involvement may be developed by specific or nonspecific exogenous antigens in genetically susceptible individuals. According to Schwaiger et al., ${ }^{31}$ blockage of cytotoxic T-lymphocyte-associated protein 4-which is one of the most potent attenuators of T-cell responses-in MRL/ $\mathrm{Mp}$ mice suppressed regulatory $\mathrm{T}$ cell function and raised the effector $\mathrm{T}$ cell response with subsequent histomorphological organ destruction similar to AIP. Freitag et al. ${ }^{29}$ described animal model with adoptive T cell transfer for AIP in HLA DRB1 0405 mice. In their study, HLA DQ8 mice did not show any chronic inflammation similar to AIP. ${ }^{29}$ They suggested HLA DQ8 may be a protective factor for the development of AIP. In our previous clinical study, no significant association between HLA class I and II and AIP was seen. ${ }^{32}$ Therefore, it remains to be determined whether our model is relevant to the human type 1 AIP.

In our previous study, ${ }^{32}$ the substitution of aspartate to other amino acids at the 57th codon of DQB1 locus appeared to represent a key genetic factor for relapse of AIP. In CD, the substitution of the 57th aspartate codon may create a positively charged P9 pocket, which confers a preference for negatively charged peptides. This DQB1 57 polymorphism has been reported to promote the recruitment of T-cell receptors bearing a negative charge in the complementary determining region 3b (CDR3B) possibly creating an abnormal high reactivity to native gluten peptides presented by HLA DQ8. ${ }^{14}$ We think that this speculation might explain a possible role of HLA-DQ8 in amplifying T-cell response against dietary gluten in AIP and $\mathrm{CD}{ }^{14}$

Disease susceptibility factors may vary among different ethnic groups and geographical regions. This diversity suggests that disease-triggering antigen may differ depending on certain genetic polymorphism. ${ }^{17}$ In this sense, our model based on HLA DQ8 mice may mimic human AIP more closely than Freitag et al.'s ${ }^{29}$ model where only interstitial pneumonitis was found as an extrapancreatic involvement. Taken together, human AIP may be a heterogeneous group with various antigens.

The environmental factors that trigger most autoimmune diseases remain unknown. One exception is $\mathrm{CD}$, which is one of the most common autoimmune disorders. ${ }^{13}$ Even though AIP responds well to steroid, relapse is common. ${ }^{32,33}$ Our study suggests that a gluten-free diet may prevent the development and relapse of AIP and/or extrapancreatic involvement in genetically susceptible individuals.

In summary, gliadin sensitization and challenge together with cerulein hyperstimulation in transgenic DQ8 mice showed prominent pancreatitis and extrapancreatic inflammation similar to human type 1 AIP. Future human clinical studies, which aim at the prevention of pancreatitis and extrapancreatic inflammation by the use of gluten-free diet in genetically suscep- 
tible individuals with HLA DQ8 allele, are required.

\section{CONFLICTS OF INTEREST}

No potential conflict of interest relevant to this article was reported.

\section{REFERENCES}

1. Sollid LM, Lie BA. Celiac disease genetics: current concepts and practical applications. Clin Gastroenterol Hepatol 2005;3:843-851.

2. Rostom A, Murray JA, Kagnoff MF. American Gastroenterological Association (AGA) Institute technical review on the diagnosis and management of celiac disease. Gastroenterology 2006;131:19812002.

3. Ansaldi N, Oderda G. Exocrine pancreatic insufficiency in celiac sprue. Gastroenterology 1981;80:883.

4. Barera G, Bazzigaluppi E, Viscardi M, et al. Macroamylasemia attributable to gluten-related amylase autoantibodies: a case report. Pediatrics 2001;107:E93.

5. Carroccio A, Iacono G, Montalto G, et al. Exocrine pancreatic function in children with coeliac disease before and after a gluten free diet. Gut 1991;32:796-799.

6. Fernanez LB, De Paula A, Prizont R, et al. Exocrine pancreas insufficiency secondary to glutenenteropathy. Am J Gastroenterol 1970;53:564-569.

7. Leeds JS, Hopper AD, Hurlstone DP, et al. Is exocrine pancreatic insufficiency in adult coeliac disease a cause of persisting symptoms? Aliment Pharmacol Ther 2007;25:265-271.

8. Ludvigsson JF, Montgomery SM, Ekbom A. Risk of pancreatitis in 14,000 individuals with celiac disease. Clin Gastroenterol Hepatol 2007;5:1347-1353.

9. Patel RS, Johlin FC Jr, Murray JA. Celiac disease and recurrent pancreatitis. Gastrointest Endosc 1999;50:823-827.

10. Pitchumoni CS, Thomas E, Balthazar E, Sherling B. Chronic calcific pancreatitis in association with celiac disease. Am J Gastroenterol 1977;68:358-361.

11. Rana SS, Bhasin DK, Sinha SK, Singh K. Coexistence of chronic calcific pancreatitis and celiac disease. Indian J Gastroenterol 2007;26:150

12. Regan PT, DiMagno EP. Exocrine pancreatic insufficiency in celiac sprue: a cause of treatment failure. Gastroenterology 1980;78:484487.

13. Galipeau HJ, Rulli NE, Jury J, et al. Sensitization to gliadin induces moderate enteropathy and insulitis in nonobese diabetic-DQ8 mice. J Immunol 2011;187:4338-4346.

14. Hovhannisyan Z, Weiss A, Martin A, et al. The role of HLA-DQ8 beta57 polymorphism in the anti-gluten T-cell response in coeliac disease. Nature 2008;456:534-538.

15. Sarles H, Sarles JC, Muratore R, Guien C. Chronic inflammatory sclerosis of the pancreas: an autonomous pancreatic disease? Am
J Dig Dis 1961;6:688-698.

16. Yoshida K, Toki F, Takeuchi T, Watanabe S, Shiratori K, Hayashi $\mathrm{N}$. Chronic pancreatitis caused by an autoimmune abnormality: proposal of the concept of autoimmune pancreatitis. Dig Dis Sci 1995;40:1561-1568.

17. Park DH, Kim MH, Chari ST. Recent advances in autoimmune pancreatitis. Gut 2009;58:1680-1689.

18. Leeds JS, Sanders DS. Risk of pancreatitis in patients with celiac disease: is autoimmune pancreatitis a biologically plausible mechanism? Clin Gastroenterol Hepatol 2008;6:951.

19. Bruhns P, Iannascoli B, England P, et al. Specificity and affinity of human Fcgamma receptors and their polymorphic variants for human IgG subclasses. Blood 2009;113:3716-3725.

20. Strait RT, Posgai MT, Mahler A, et al. IgG1 protects against renal disease in a mouse model of cryoglobulinaemia. Nature 2015;517:501-504.

21. Demols A, Van Laethem JL, Quertinmont E, et al. Endogenous interleukin-10 modulates fibrosis and regeneration in experimental chronic pancreatitis. Am J Physiol Gastrointest Liver Physiol 2002;282:G1105-G1112.

22. Brooks SE, Golden MH. The exocrine pancreas in kwashiorkor and marasmus: light and electron microscopy. West Indian Med J 1992;41:56-60.

23. Nousia-Arvanitakis S, Karagiozoglou-Lamboudes T, Aggouridaki C, Malaka-Lambrellis E, Galli-Tsinopoulou A, Xefteri M. Influence of jejunal morphology changes on exocrine pancreatic function in celiac disease. J Pediatr Gastroenterol Nutr 1999;29:81-85.

24. Pietruczuk M, Dabrowska MI, Wereszczynska-Siemiatkowska U, Dabrowski A. Alteration of peripheral blood lymphocyte subsets in acute pancreatitis. World J Gastroenterol 2006;12:5344-5351.

25. Salvati VM, MacDonald TT, Bajaj-Elliott M, et al. Interleukin 18 and associated markers of $\mathrm{T}$ helper cell type 1 activity in coeliac disease. Gut 2002;50:186-190.

26. Sadr-Azodi 0, Sanders DS, Murray JA, Ludvigsson JF. Patients with celiac disease have an increased risk for pancreatitis. Clin Gastroenterol Hepatol 2012;10:1136-1142.e3.

27. Harris LA, Park JY, Voltaggio L, Lam-Himlin D. Celiac disease: clinical, endoscopic, and histopathologic review. Gastrointest Endosc 2012;76:625-640.

28. Kawa S, Ota M, Yoshizawa K, et al. HLA DRB10405-DQB10401 haplotype is associated with autoimmune pancreatitis in the Japanese population. Gastroenterology 2002;122:1264-1269.

29. Freitag TL, Cham C, Sung HH, et al. Human risk allele HLADRB1*0405 predisposes class II transgenic Ab0 NOD mice to autoimmune pancreatitis. Gastroenterology 2010;139:281-291.

30. Aalberse RC, Stapel SO, Schuurman J, Rispens T. Immunoglobulin G4: an odd antibody. Clin Exp Allergy 2009;39:469-477.

31. Schwaiger T, van den Brandt C, Fitzner B, et al. Autoimmune pancreatitis in MRL/Mp mice is a T cell-mediated disease responsive to cyclosporine A and rapamycin treatment. Gut 2014;63:494-505.

32. Park do H, Kim MH, Oh HB, et al. Substitution of aspartic acid at 
850 Gut and Liver, Vol. 10, No. 5, September 2016

position 57 of the DQbeta1 affects relapse of autoimmune pancreatitis. Gastroenterology 2008;134:440-446.

33. Hart PA, Kamisawa T, Brugge WR, et al. Long-term outcomes of autoimmune pancreatitis: a multicentre, international analysis. Gut 2013;62:1771-1776. 